\title{
Functional Nanomaterials and their 2D and 3D Fabrications for Drug Delivery Applications
}

\author{
Wei-Fan Lee \\ Grade 10, Taipei Wego Senior High School, Taiwan
}

\begin{abstract}
This review paper would be discussing both the manufacture of the functional nanomaterials and their practical applications for drug delivery The study of nanomaterials has been a coruscating field of research that has a huge impact on science, engineering, and medicine. In terms of applied science, it succeeded the limits of conventional materials, hence making the opt of the materials more multifaceted. Speaking of drug delivery, new research on nanomaterials marked an outset of a new study of medicine and might have changed our perception of drugs. Nanomaterials are recognized to be a emerging material with good prospect for drug delivery regarding their particular characteristics. Because of these benefits, further research on this topic is conducted as of now.
\end{abstract}

\section{Introduction}

\subsection{Importance of Gold NPs}

In the field of drug delivery, NPs are widely used because of their unique properties. Often, NPs are functionalized to meet the requirement for different usages. Among the functionalized NPs, gold particles is one of the best choices among the functionalized NPs because it serves as an excellent agent of carrying anti-cancer during the cancer therapy, and many other significant advantages. It also serves as a dose enhancer during radiotherapy. As Figure 1 shows, the functions of Au NPs can be easily altered by attaching the NP to different substances. This shows that Au NPs are advantageous because of their wide usage.

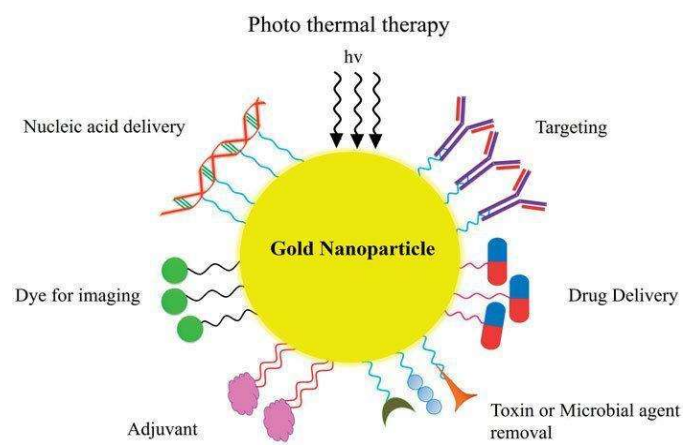

Figure 1 Various functions of Au NPs by altering the materials attached to the NP [1]

As of now, thanks to the advancements in technology, producers now has the ability to fabricate different size, composition, morphology, or other properties by applying precise technology [2]. The chart below demonstrates the variation of $\mathrm{Au}$ NPs in terms of properties [2].
Table 1 Variety of properties of Au NPs

\begin{tabular}{|c|c|c|c|c|}
\hline $\begin{array}{l}\text { Diamet } \\
\text { er }(n m)\end{array}$ & $\begin{array}{c}\text { Nanopartic } \\
\text { les } / \mathrm{mL}\end{array}$ & $\begin{array}{c}\text { Peak SPR } \\
\text { Waveleng } \\
\text { th }(\mathrm{nm})\end{array}$ & $\begin{array}{c}\text { Mol } \\
\text { ar } \\
\text { Ext } \\
\left(\mathrm{M}^{-}\right. \\
{ }^{1} \cdot \mathrm{cm}^{-} \\
\left.{ }^{1}\right)\end{array}$ & $\begin{array}{c}\text { Product } \\
\text { Numbers } \\
\text { (Surfacta } \\
\text { nt } \\
\text { Stabilize } \\
\text { d) }\end{array}$ \\
\hline 5 & $5.47 \times 10^{13}$ & $515-520$ & $\begin{array}{r}1.10 \\
\times 10^{7}\end{array}$ & 741949 \\
\hline 10 & $5.98 \times 10^{12}$ & $515-520$ & $\begin{array}{c}1.01 \\
\times 10^{8}\end{array}$ & 741957 \\
\hline 15 & $1.64 \times 10^{12}$ & 520 & $\begin{array}{l}3.67 \\
\times 10^{8}\end{array}$ & 777137 \\
\hline 20 & $6.54 \times 10^{11}$ & 524 & $\begin{array}{l}9.21 \\
\times 10^{8}\end{array}$ & 741965 \\
\hline 30 & $1.79 \times 10^{11}$ & 526 & $\begin{array}{l}3.36 \\
\times 10^{9}\end{array}$ & 741973 \\
\hline 40 & $7.15 \times 10^{10}$ & 530 & $\begin{array}{c}8.42 \\
\times 10^{9}\end{array}$ & 741981 \\
\hline 50 & $3.51 \times 10^{10}$ & 535 & $\begin{array}{c}1.72 \\
x \\
10^{10}\end{array}$ & 742007 \\
\hline 60 & $1.96 \times 10^{10}$ & 540 & $\begin{array}{c}3.07 \\
\mathrm{x}\end{array}$ & 742015 \\
\hline
\end{tabular}




\begin{tabular}{|c|c|c|c|l|}
\hline & & & $10^{10}$ & \\
\hline 80 & $7.82 \times 10^{9}$ & 553 & $\begin{array}{c}7.70 \\
\times\end{array}$ & 742023 \\
\hline 100 & $3.84 \times 10^{10}$ & 572 & $\begin{array}{c}1.57 \\
\times \\
10^{11}\end{array}$ & 742031 \\
\hline 150 & $3.60 \times 10^{9}$ & $\begin{array}{c}\text { Not } \\
\text { Measured }\end{array}$ & N/A & 742058 \\
\hline 200 & $1.9 \times 10^{9}$ & $\begin{array}{c}\text { Not } \\
\text { Measured }\end{array}$ & N/A & 742066 \\
\hline 250 & $7.1 \times 10^{8}$ & $\begin{array}{c}\text { Not } \\
\text { Measured }\end{array}$ & N/A & 742074 \\
\hline 300 & $4.5 \times 10^{8}$ & $\begin{array}{c}\text { Not } \\
\text { Measured }\end{array}$ & N/A & 742082 \\
\hline 400 & $1.9 \times 10^{8}$ & $\begin{array}{c}\text { Not } \\
\text { Measured }\end{array}$ & N/A & 742090 \\
\hline
\end{tabular}

\subsection{Biodegradable nanoparticles}

New research in nanotechnology allowed NPs to be applied to medicine. They became an important aspect of therapy since it is potentially beneficial for creating personalized medicine [2]. Among the NPs, biodegradable NPs are vital for the field of drug delivery, since they can be directly consumed without toxicity to the body. Biodegradable NPs like polylactide (PLA) allowed the medicine to be enclosed in the matrix, and because of its small size relative to the cells, NPs are able to travel through the membranes of the cells [44].

\subsection{D nanomaterials in Drug delivery}

Graphene, a new 2D nanomaterial discovered in 2005 [3], is one of the nanomaterials with good prospects. Since the carbon atoms in graphene are arranged in numerous hexagons (honeycomb shape) through hybridization bonds, it is able to perform numerous properties. Currently, 2D nanomaterial would be a scintillating new era for the field of drug delivery. Both its physical properties (e.g. size, width) and chemical properties would have a notable impact on the field of drug delivery [43].

\section{Gold Nanoparticle}

\subsection{Properties of Gold Nanoparticles}

Gold nanoparticle (Au NPs) has been widely utilized in the field of biotechnology because of its biocompatibility and its several surface functionalities [4]. (Figure 2) They are also beneficial because they can serve as effective radiosensitizers. This property can be extremely vital in the field of drug delivery [5]. Spherical Au NPs have beneficial properties as large surface-to-volume ratio, low toxicity [4].

\subsection{Gold nanoparticles-Based Drug Delivery}

$\mathrm{Au}$ NPs based drug delivery has been one of the scintillating fields of drug delivery regarding its superior performance. Albeit there is no pharmaceutical approval of $\mathrm{Au}$ NPs based nanodrugs, much research in Au NPs based drug delivery has been conducted [7]. Au NPs are recognized as effective nano carriers for various kinds of drugs. (Figure 3)

Fucoidan, for reference, is one of immune-therapeutic function polymers that is mostly utilized in pharmaceutical applications. Au NPs that is synthesized with fucoidan perform even better biocompatibility and nontoxicity than those synthesized with naturally occurring polymers (e.g. chitosan, porphyran).

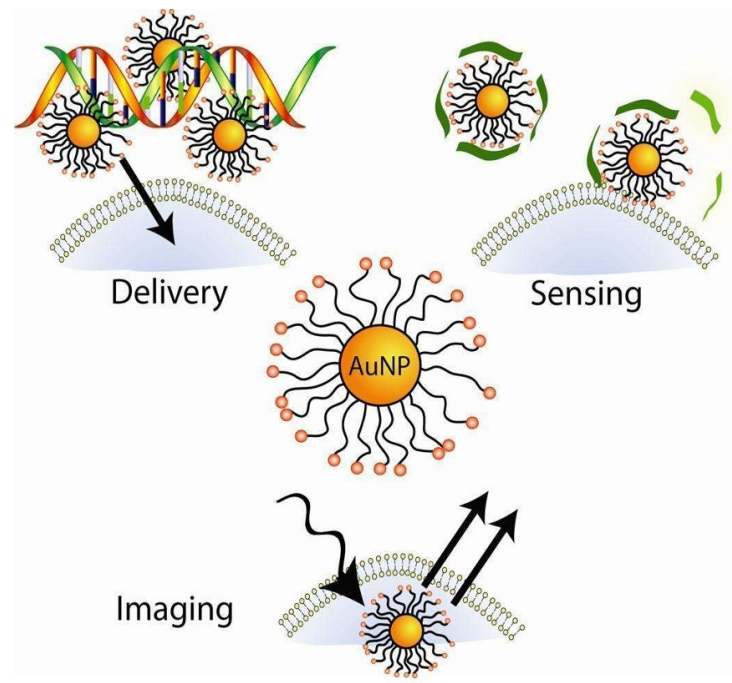

Figure 2 Application of Au NPs in biotechnology [6] Chemotherapeutical

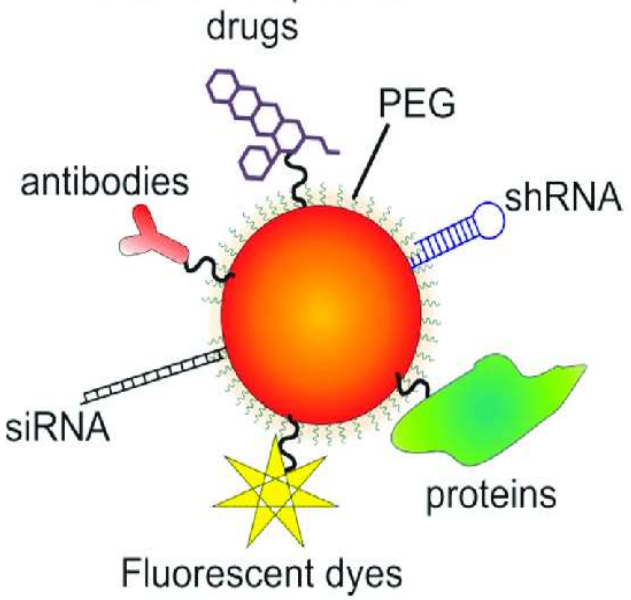

Figure 3 Au NPs functionalization [10]

\section{Two-dimensional nanomaterials for drug delivery}

The process of drug delivery requires particles, which usually comprise lipids, polymers, or other materials, to 
combine with the therapeutics to substitute the conventional drugs. The free drug system can confront difficulties such as low solubility, loss of drug activity in particular situations. Nano drug delivery focuses on establishing bioactive and biocompatible molecules. Thus, drug delivery systems are expected to evenly release drugs at a designated time [4].

\subsection{Graphene}

Graphene is a 2D, flat monolayer carbon atoms in the shape of a honeycomb. (Figure 4) It is the basic unit of other graphitic structure (e.g., fullerene (0D), carbon nanotube (1D), and graphite (3D)). Graphene is applied in many research fields due to its advantageous properties (e.g. electric, thermal, optical, magnetic). In the study of biological applications, graphene is also beneficial because of its simple biofunctionalization [4] [11].

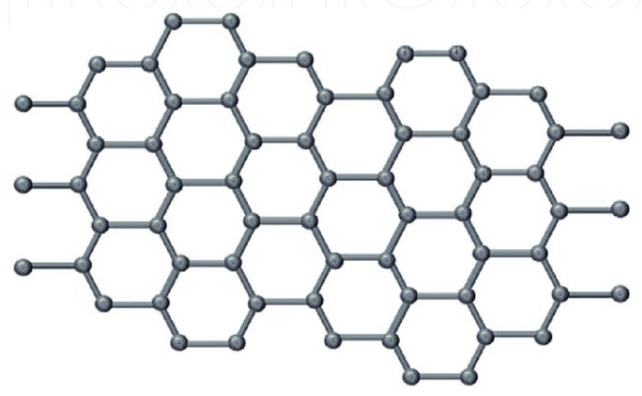

Figure 4 Structure of graphene. Reproduced from [11]

In terms of drug delivery, graphene nanomaterials (GNMs) can be highly beneficial because of their properties (e.g. surface area, layer numbers, lateral dimensions) [12]. Moreover, graphene responds to many environmental factors, including magnetic and electric field, and the existence of enzyme.

\subsection{Graphene oxide (GO)}

After the oxidation of graphene, numbers of functional group (e.g. epoxide, hydroxyl, carboxylic acid), can be observed on the surface of GO [13]. After the oxidation process, its biocompatibility and combination with biomolecules are enhanced, making it a potential material to be applied to drug delivery [14]. In general, Hummer's method (Figure 8) is usually recognized as the best preparation method of GO since the hydroxyl and epoxide groups can perform a strong $\pi-\pi$ bond for drug loading [15].

After synthesis, numerous functional groups (e.g. epoxide, hydroxyl) will be seen on the surface, which can then be used for various purposes [16]. The list below organizes the recent research in the functionalization of GO.
Table 2 Recent research in the functionalization of GO.

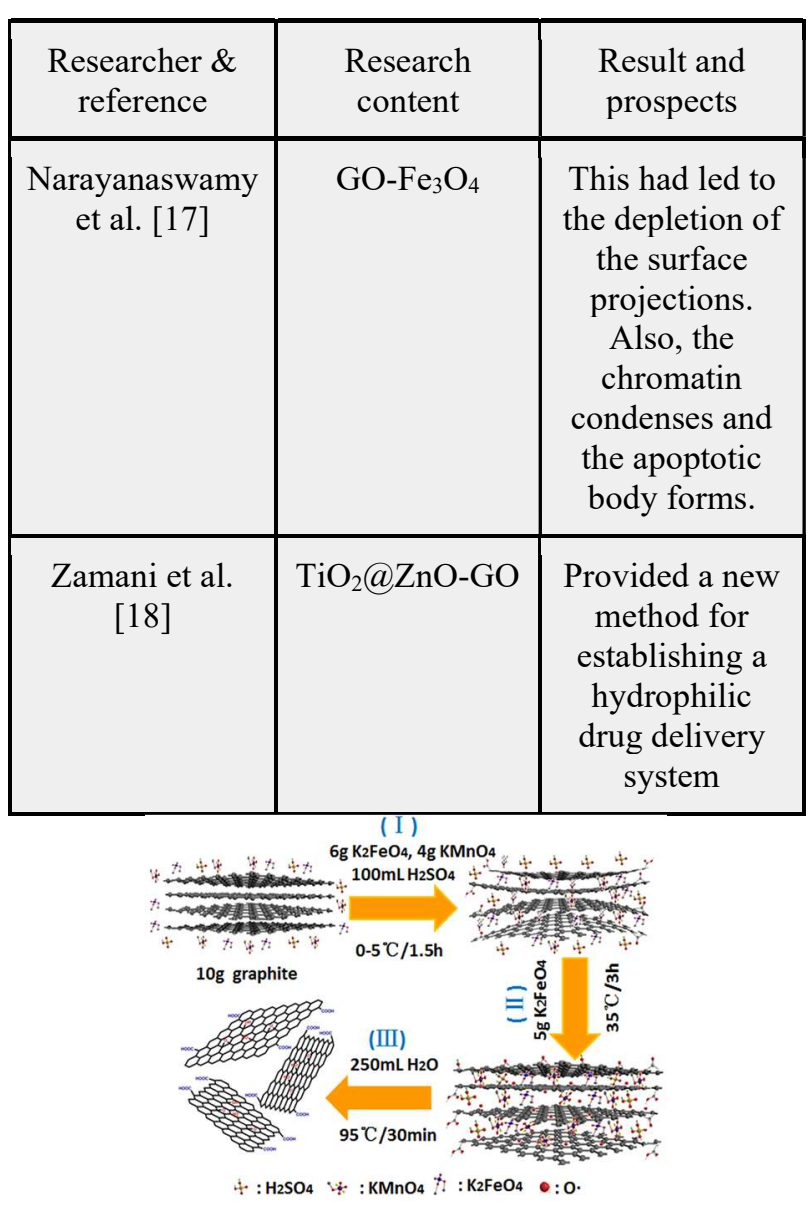

Figure 5 Hummer's method for fabricating GO

\subsection{Black Phosphorus}

Black phosphorus (BP), another emerging nanomaterial, has the advantage of switchable band gaps, extinct biodegradability, which may be a potential material used for loading biomolecules, drugs, or antibodies [4,21].

BP nanosheets are usually fabricated by exfoliating from BP crystals, which possesses strong interaction between phosphorus and comparatively weak van der Walls force [22]. Its hexagonal honeycomb-like arrangement of the atoms (Figure 9) gives it a semiconductor nature, superior light absorption capability [23] [24]. (a)

(b)

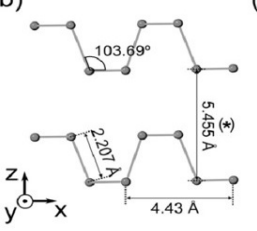

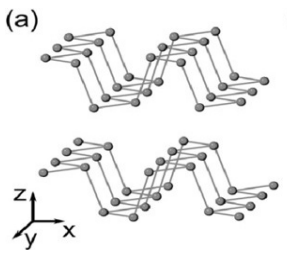

(c)

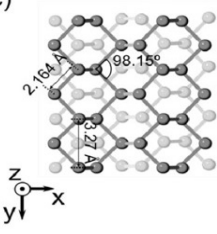

Figure 6 Physical arrangement of BP

$\mathrm{BP}$ is rather significant among the nanomaterials used for drug delivery systems (DDSs) due to its high SA/V, photosensitivity, superior biodegradability, and biocompatibility. As shown in Figure 10, there are several categories of BP platforms used for DDSs, including BPN, BPQDs, BPHs [25]. BP has been proving itself to be an 
effective carrier of drugs, RNA interference (siRNA), inorganic substances (e.g., UCNP, Pt) [25].

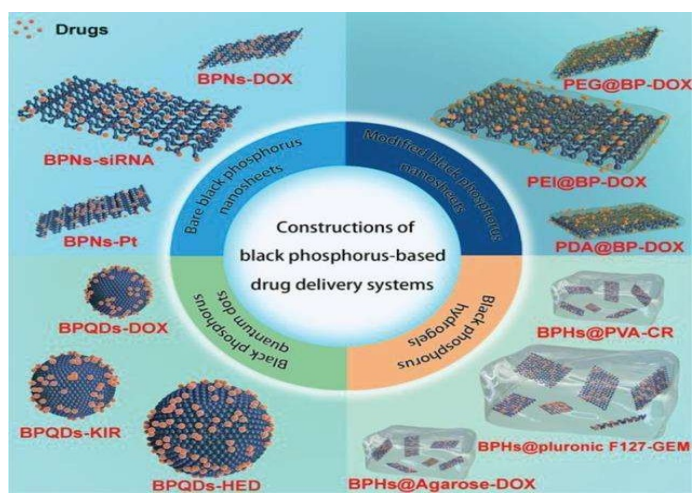

Figure 7 Categories of BP platforms

\subsection{Transitional Metal Dichalcogenides (TMDs)}

TMDs are a unique kind of compound that is frequently utilized in the field of nanotechnology. Its formula can be presented as $\mathrm{MX}_{2}$, where $\mathrm{M}$ symbolizes the transitional metals, while $\mathrm{X}$ represents the chalcogen. The structure of the TMDs usually varies because of different coordination spheres of the metal atoms, which allows diverse loading capability [26]. Conventional TMDs (e.g. $\mathrm{MoS}_{2}, \mathrm{WS}_{2}$, $\mathrm{MoSe}_{2}, \mathrm{WSe}_{2}$ ), performs the unique structure of planar TMDs crystals.

Thin layer TMDs possess several advantageous attributes which make them potential candidates for the DDSs [27]. In some cases, TMDs perform even more advantageous properties than other 2D nanomaterials. For example, 2D $\mathrm{MoS}_{2}$ had been proven to have higher loading capacity than GO because of its surface absorption effect [28].

\subsection{Transitional Metal Oxides (TMOs)}

TMOs are the compound that comprises transitional metal and oxygen. With their $2 \mathrm{p}$ metal being filled, TMOs owns a nature of having valence band. The conduction bands can be an important aspect of determining its electric and magnetic properties, which is vital for its application in photochromic and thermochromic related fields [29]. Among the TMOs, several materials as $\mathrm{MnO}_{2}, \mathrm{TiO}_{2}, \mathrm{ZnO}$ stands out especially since they perform unique ferromagnetic and redox properties, which may be advantageous for constructing photo-sensitizers for DDSs [29].

The redox characteristics of $\mathrm{MnO}_{2} 2 \mathrm{D}$ nanosheets gives it a signal-responsive nature. With that being said, it is one of the promising candidates to be utilized in cancer therapy [29]. The redox/pH controllable PTX DDSs developed by Choi and colleagues had been proven to reduce many side effects, which can be important for the field of cancer therapy [4].

$\mathrm{TiO}_{2} \mathrm{NPs}$ are also a kind of rising materials because of its semiconductive nature with a wide bandgap [4]. With regard to its $2 \mathrm{D}$ characteristic, $\mathrm{TiO}_{2}$ also has a superior loading capacity. Research conducted by Kushnirov and Sosnik [31] had successfully realized the fabrication of $\mathrm{TiO}_{2} /$ polymer amphiphilic NPs with controllable size and outstanding performances in DDSs.

\subsection{Transition metal carbides (TMCs)}

Transitional metals carbides (TMCs) are compounds composed of early transitional metals (e.g. Ti, Mo, V, Nb), and $\mathrm{C}$ or $\mathrm{N}$ [29]. MXenes are potential candidates in many fields including diagnostic imaging, biosafety, issues, making MXenes one of the pioneering nanomaterials among all [33]. As other 2D nanomaterials, MXenes can be functionalized in order to apply it for cancer therapy or other field of application.

Just as TMOs, TMCs also have a large loading capacity of various biomolecules. Research conducted by Han and colleagues had successfully demonstrated that TMCs can effectively load various kinds of organic dyes. To better demonstrate the property of TMCs, Chen and coworkers evaluated its loading capacity, $\mathrm{pH} / \mathrm{NIR}$-triggered release of drugs, PA imaging ability [34]. Results demonstrated the biosafety of MXenes by proving that it can be naturally emitted after a particular time.

\section{Types of Biodegradable Nanoparticles and their applications in nanomedicines}

\subsection{Biodegradable Nanoparticles in targeted Delivery System}

Biodegradable NPs used in targeted DDSs can be categorized in regard to their structures or their arrangements just as the nonbiodegradable substances. The wanted substance can either be encapsulated or attached onto the surface of the biodegradable NPs [34]. In general biodegradable NPs possess the conventional advantages NPs like slow and controllable release of drugs. Just as other nanomaterials, biodegradable NPs can be further functionalized to meet different demands [34]. During the process of synthesis, polymers should have natures of biocompatibility and biosafety. Biodegradable NPs, on the other hand, are usually colloidal particles that is available of combining with functional groups. Based on the polymers, polymer-based nanomaterials can be categorized by examining if it is synthesized or natural.

\subsection{Antioxidant Delivery}

Oxidation form of substances has been associated with the hazardous effects on cells[36]. If the DDSs could tackle with the issue by delivering agents counters oxidative stress, cell wound given rise to by reactive oxygen species (ROS) can be relieved to a certain extent [37].

To realize the idea of antioxidant delivery, Kang and coworker [38] established a new NP, namely PVAX, a substance that comprises copolyoxalate with vanillyl alcohol (VA). When PVAX confronts relatively high concentration of $\mathrm{H}_{2} \mathrm{O}_{2}$, PVAX would degrade and then release VA, which can serve as an anti-inflammatory and anti-apoptotic substance.

\subsection{Oral Drug Delivery}

Comparative to injection, orally consumed drug can largely decrease the probability of getting infection and physical discomfort, especially to the diabetic patients [35]. $1.6 \% \mathrm{Zn}$ insulin in PLGA, for reference, was 
developed a decade ago to realize the idea of oral drug delivery. Although the practicality of $\mathrm{Zn}$ insulin was relatively low, it still paved the way for the future research. Research in oral drug delivery is still being conducted to this day.

\section{Conclusion}

The article aforementioned had arranged the organized the recent research in functional nanomaterials and their 2D and 3D fabrications for DDSs application. From these topics, one can analyze the vital attributes for nanomaterials to be applied in biotechnology include distinct biocompatibility, biodegradability, noncytotoxicity.

As of now, nanomaterials have been paving a path for research in many fields. For DDSs, nanomaterials are rising materials to be researched furthermore because of their extinct attributes. The current research progress would not be an terminal to the development of nanomaterials; rather, it marked the outset of another era of DDSs.

\section{References}

1. Using gold nanoparticles in diagnosis and treatment of melanoma cancer - Scientific Figure on ResearchGate. Available from:

https://www.researchgate.net/figure/Differentapplications-of-gold-nanoparticles-in-diagnosis-andtherapy-Nanoparticles-are_fig3_322739738 [accessed 5 Nov, 2021]

2. Gold Nanoparticles: Properties and Applications, www.sigmaaldrich.com/TW/en/technicaldocuments/technical-article/materials-science-andengineering/biosensors-and-imaging/goldnanoparticles.

3. Gold Nanoparticles: Properties and Applications, www.sigmaaldrich.com/TW/en/technicaldocuments/technical-article/materials-science-andengineering/biosensors-and-imaging/goldnanoparticles.

4. Mei, Xuan, et al. "Recent advancements in twodimensional nanomaterials for drug delivery." Wiley Interdisciplinary Reviews: Nanomedicine and Nanobiotechnology 12.2 (2020): e1596.

5. Yeh, Yi-Cheun, et al. "Gold Nanoparticles: Preparation, Properties, and Applications in Bionanotechnology." Nanoscale, U.S. National Library of Medicine, 21 Mar. 2012, www.ncbi.nlm.nih.gov/pmc/articles/PMC4101904/.

6. Siddique, Sarkar, and James C. Chow. "Gold Nanoparticles for Drug Delivery and Cancer Therapy." Applied Sciences, vol. 10, no. 11, 2020, p. 3824., doi:10.3390/app10113824.

7. Farooq, Muhammad U., et al. "Gold NanoparticlesEnabled Efficient Dual Delivery of Anticancer Therapeutics to Hela Cells." Nature News, Nature
Publishing Group, 13 Feb. 2018, www.nature.com/articles/s41598-018-21331-y.

8. Jeong, Eun Hye, et al. "Gold Nanoparticle (Aunp)Based Drug Delivery and Molecular Imaging for Biomedical Applications." Archives of Pharmacal Research, vol. 37, no. 1, 2013, pp. 53-59., https://doi.org/10.1007/s12272-013-0273-5.

9. Tumor Microenvironment Modulation via Gold Nanoparticles Targeting Malicious Exosomes: Implications for Cancer Diagnostics and Therapy Scientific Figure on ResearchGate. Available from https://www.researchgate.net/figure/Goldnanoparticles-AuNPs-functionalization-fortheranostics-After-

functionalization_fig1_312481625 [accessed 30 Oct, 2021]

10. Bai, Renu Geetha, and Ghaleb A. Husseini. "Graphene-Based Drug Delivery Systems." Biomimetic Nanoengineered Materials for Advanced Drug Delivery, 2019, pp. 149-168., https://doi.org/10.1016/b978-0-12-814944-7.000114.

11. Graphene: Fabrication Methods, Properties, and ApplicatioZns in Modern Industries - Scientific Figure on ResearchGate. Available from: https://www.researchgate.net/figure/Basic-structureof-graphene-6_fig1_340942598 [accessed 24 Oct, 2021]

12. Liu, Jingquan, et al. "Graphene and Graphene Oxide as New Nanocarriers for Drug Delivery Applications." Acta Biomaterialia, vol. 9, no. 12, 2013, pp. 92439257., https://doi.org/10.1016/j.actbio.2013.08.016.

13. Cai, Ren, et al. "Fabrication of Ultrathin $\mathrm{Zn}(\mathrm{Oh}) 2$ Nanosheets as Drug Carriers." Nano Research, vol. 9, no. 8, 2016, pp. 2520-2530., https://doi.org/10.1007/s12274-016-1138-2.

14. Sharafeldin, Mohamed, et al. "FE3O4 Nanoparticles on Graphene Oxide Sheets for Isolation and Ultrasensitive Amperometric Detection of Cancer Biomarker Proteins." Biosensors and Bioelectronics, vol. 91, 2017, pp. 359-366., https://doi.org/10.1016/j.bios.2016.12.052.

15. Yang, Lingyan, et al. "Photothermal Therapeutic Response of Cancer Cells to Aptamer-Gold Nanoparticle-Hybridized Graphene Oxide under Nir Illumination." ACS Applied Materials \&amp; Interfaces, vol. 7, no. 9, 2015, pp. 5097-5106., https://doi.org/10.1021/am508117e.

16. Narayanaswamy, Venkatesha, et al. "Go-FE3O4 Nanoparticle Composite for Selective Targeting of Cancer Cells." Nano Biomedicine and Engineering, vol. 9, no. 2017, https://doi.org/10.5101/nbe.v9i1.p96-102.

17. Zamani, Mostafa, et al. "Mesoporous Titanium Dioxide@Zinc Oxide-Graphene Oxide Nanocarriers for Colon-Specific Drug Delivery." Journal of Materials Science, vol. 53, no. 3, 2017, pp. 16341645., https://doi.org/10.1007/s10853-017-1673-6. 
18. Wang, Hui, et al. "Ultrathin Black Phosphorus Nanosheets for Efficient Singlet Oxygen Generation." Journal of the American Chemical Society, vol. 137, no. 35, 2015, pp. 11376-11382., https://doi.org/10.1021/jacs.5b06025.

19. Yu, Huitao, et al. "High-Efficient Synthesis of Graphene Oxide Based on Improved Hummers Method." Scientific Reports, vol. 6, no. 1, 2016, https://doi.org/10.1038/srep36143.

20. Wang, Huaiyu, and Xue-Feng Yu. "Few-Layered Black Phosphorus: From Fabrication and Customization to Biomedical Applications." Small, vol. 14, no. 6, 2017, p. 1702830., https://doi.org/10.1002/smll.201702830.

21. $\mathrm{Hu}, \mathrm{Guohua}$, et al. "Black Phosphorus Ink Formulation for Inkjet Printing of Optoelectronics and Photonics." Nature Communications, vol. 8, no. 1, 2017, https://doi.org/10.1038/s41467-017-003581.

22. Isolation and characterization of few-layer black phosphorus - Scientific Figure on ResearchGate. Available from: https://www.researchgate.net/figure/Blackphosphorus-structure-Schematic-diagram-of-thecrystalline-structure-of-black_fig8_260483012 [accessed 31 Oct, 2021]

23. Liu, Wenxin, et al. "Current Advances in Black Phosphorus - Based Drug Delivery Systems for Cancer Therapy." Advanced Science, vol. 8, no. 5, 2021, p. https://doi.org/10.1002/advs.202003033.

24. Li, Likai, et al. "Quantum Hall Effect in Black Phosphorus Two-Dimensional Electron System." Nature Nanotechnology, vol. 11, no. 7, 2016, pp. 593-597., https://doi.org/10.1038/nnano.2016.42.

25. Mak, Kin Fai, and Jie Shan. "Photonics and Optoelectronics of 2D Semiconductor Transition Metal Dichalcogenides." Nature Photonics, vol. 10, no. 4, 2016, pp. 216-226., https://doi.org/10.1038/nphoton.2015.282.

26. Anju, S., and PV. Mohanan. "Biomedical Applications of Transition Metal Dichalcogenides (Tmdes)." Synthetic Metals, vol. 271, 2021, p. 116610 , https://doi.org/10.1016/j.synthmet.2020.116610.

27. Tian, Bo, et al. "Photothermally Enhanced Photodynamic Therapy Delivered by NanoGraphene Oxide." ACS Nano, vol. 5, no. 9, 2011, pp. 7000-7009., https://doi.org/10.1021/nn201560b.

28. Song, Fang, et al. "Transition Metal Oxides as Electrocatalysts for the Oxygen Evolution Reaction in Alkaline Solutions: An Application-Inspired Renaissance." Journal of the American Chemical Society, vol. 140, no. 25, 2018, pp. 7748-7759., https://doi.org/10.1021/jacs.8b04546.

29. Choi, G., Piao, H., Alothman, Z. A., Vinu, A., Yun, C.-O., \& Choy, J.-H. (2016). Anionic clay as the drug delivery vehicle: Tumor targeting function of layered double hydroxide-methotrexate nanohybrid in C33A orthotopic cervical cancer model. International Journal of Nanomedicine, 11, 337-348.

30. Kulkarni, M., Mazare, A., Gongadze, E., Perutkova, Š., Kralj-Iglič, V., Milošev, I., ... Mozetičn, M. (2015). Titanium nanostructures for biomedical applications. Nanotechnology, 26, 062002.

31. Kushnirov Melnitzer, Vladislav, and Alejandro Sosnik. "Hybrid Titanium Oxide/Polymer Amphiphilic Nanomaterials with Controlled Size for Drug Encapsulation and Delivery." Advanced Functional Materials, vol. 30, no. 18, 2018, p. 1806146., https://doi.org/10.1002/adfm.201806146.

32. Soleymaniha, Mohammadreza, et al. "Promoting Role of Mxene Nanosheets in Biomedical Sciences: Therapeutic and Biosensing Innovations." Advanced Healthcare Materials, vol. 8, no. 1, 2018, p. 1801137. https://doi.org/10.1002/adhm.201801137.

33. Lin, Han, et al. "Two-Dimensional Ultrathin Mxene Ceramic Nanosheets for Photothermal Conversion." Nano Letters, vol. 17, no. 1, 2016, pp. 384-391., https://doi.org/10.1021/acs.nanolett.6b04339.

34. Su, Shi, and Peter M. Kang. "Systemic Review of Biodegradable Nanomaterials in Nanomedicine." Nanomaterials, vol. 10, no. 4, 2020, p. 656., https://doi.org/10.3390/nano10040656.

35. Villemin, Elise, et al. "Polymer Encapsulation of Ruthenium Complexes for Biological and Medicinal Applications." Nature Reviews Chemistry, vol. 3, no. 4, 2019, pp. 261-282., https://doi.org/10.1038/s41570-019-0088-0. 38

36. Manke, Amruta, et al. "Mechanisms of NanoparticleInduced Oxidative Stress and Toxicity." BioMed Research International, vol. 2013, 2013, pp. 1-15., https://doi.org/10.1155/2013/942916.

37. Lee, D.; Bae, S.; Hong, D.; Lim, H.; Yoon, J.H.; Hwang, O.; Park, S.; Ke, Q.; Khang, G.; Kang, P.M. H2O2 -Responsive Molecularly Engineered Polymer Nanoparticles as Ischemia/Reperfusion-Targeted Nanotherapeutic Agents. Sci. Rep. 2013, 3, 2233.

38. Bae, Soochan, et al. "Hydrogen Peroxide-Responsive Nanoparticle Reduces Myocardial Ischemia/Reperfusion Injury. " Journal of the American Heart Association, vol. 5, no. 11, 2016, https://doi.org/10.1161/jaha.116.003697.

39. Using gold nanoparticles in diagnosis and treatment of melanoma cancer - Scientific Figure on ResearchGate. Available from: https://www.researchgate.net/figure/Differentapplications-of-gold-nanoparticles-in-diagnosis-andtherapy-Nanoparticles-are_fig3_322739738 [accessed 5 Nov, 2021] 